\title{
The Response of Normal Shocks in Diffusers
}

\author{
F.E.C. Culick* \\ California Institute of Technology, Pasadena, California \\ and \\ T. Rogerst \\ The Marquardt Company, Van Nuys, California
}

\begin{abstract}
The frequency response of a normal shock in a diverging channel is calculated for application to problems of pressure oscillations in ramjet engines. Two limits of a linearized analysis are discussed: one represents isentropic flow on both sides of a shock wave; the other may be a crude approximation to the influence of flow separation induced by the wave. Numerical results are given, and the influences of the shock wave on oscillations in the engine are discussed.
\end{abstract}

\section{Nomenclature}

a $\quad$ speed of sound

4. $=$ admittance function, defincd by Eq. (9)

$C \quad=$ constant defined by Eq. (43)

$k \quad=$ complex wave number, $k=(\omega-i \alpha) / \bar{a}$

$M \quad=$ Mach number

$p \quad=$ pressure

$P \quad=$ defined by Eq. (13)

$P_{+}, P_{-} \quad=$ amplitudes of rightward and leftward traveling

pressure waves, respectively

$P_{\mathrm{s}} \quad=$ defined by Eq. (29)

$S=$ cross-sectional area

t $\quad=$ time

u $\quad=$ velocity

$U_{+}, U_{-}=$amplitudes of rightward and leftward traveling velocity waves, respectively

$U_{\mathrm{s}} \quad=$ defined by Eq. (30)

$v_{s} \quad=$ velocity fluctuation of normal shock

$x \quad=$ position coordinate along the axis of the duct

$x_{s}=$ position fluctuation of normal shock

$\alpha \quad=$ decay constant in space

$\beta \quad=$ reflection coefficient, defined by Eq. (10)

$\gamma \quad=$ ratio of specific heats

$\rho \quad=$ density

$\tau=$ time constant, defined by Eq. (44)

$\phi \quad=$ phase of the rcflection coefficient, defined by Eq. (18)

$\psi \quad=$ defined by Eq. (15)

$\psi^{\prime \prime} \quad$ = defined by Eq. (16)

w = angular frequency, $\mathrm{rad}^{\prime} \mathrm{s}$

$\Omega \quad=$ dimensionless angular frequency, defined by Eq. (32)

\section{Superscripts}

( ) = average value

( ) ${ }^{\prime}=$ fluctuation

Subscripts

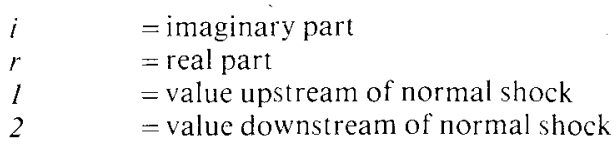

Presented as Paper 81-1431 at the AIAA/SAE/ASME 17th Joint Propulsion Conference, Colorado Springs, Colo., July 27-29, 1981; submitted May 6, 1982; revision received Jan. 26, 1983. Copyright (C) American Institutc of Aeronautics and Astronautics, Inc., 1983. All rights reserved.

*Professor, Applied Physics and Jet Propulsion. Associate Fellow AIAA.

†Senior Development Engineer. Member AIAA (Deceased).

\section{Introduction}

$\mathbf{R}$ ECENT concern!-4 with undesirable consequences of ongitudinal pressure oscillations in ramjet engines has focused attention on unsteady behavior of the inlet diffuser. In extreme cases the compressive portion of an oscillation may be so large as to cause the inlet to become unchoked. Thus, the effect of a pressure oscillation may be regarded as an equivalent loss of pressure margin.

Although the flow is surely morc complicated in actual cases, it is convenient to begin with a simplified representation of the diffuser, and assume that under steady operating conditions a single normal shock exists in the divergent section. Moreover, as a first approximation, it is in many respects quite adequate to treat the pressure oscillations within the approximations of small-amplitude acoustical motions. Thus, the problem of the behavior of the inlet comes down to a problem of interactions between a normal shock and acoustic waves

Burgers ${ }^{5}$ seems to have been the first to treat a problem of this sort. He examined the transmission and reflection of waves having phase fronts parallel to a plane shock wave moving in a uniform gas. The two cases in which acoustic waves are generated by an independent source upstream or downstream of the shock were treated. With some rewriting, the results for the sccond case can be shown to be identical with those given here for a normal shock in a channel having constant cross-sectional area.

Independently, Kantrowitz $z^{6}$ analyzed the stability of shock waves in a nonuniform channel. He proved the now familiar results that smooth deceleration of the flow through the speed of sound is unstable (i.e., occurs through a shock wave in practice), and that a normal shock is stable in a diverging channel and unstable in a converging channel. The problem of stability was treated by supposing that a shock assumed to be stationary in the channel suffers a small displacement from its initial position. This causes a disturbance to propagate downstream, which is then reflected at some station, causing a disturbance to propagate upstream to the shock. The question to be answered is: Does the disturbance given the shock ultimately grow or decay? Posed in this fashion, the problem can be solved only if a definite condition for reflection at the downstream station is specified. Three special cases were treated in Ref. 6, all giving the conclusion cited above. The analysis produced formulas for the relaxation time required for the shock to return to its equilibrium position in a divergent channel.

Essentially the same technique was used later by Hurrell ${ }^{7}$ to determine the relaxation time of a normal shock subjected to steady oscillations in a divergent channel. Although the analysis requires a cumbersome computation to satisfy the 
downstream conditions of the shock wave, the result, as we shall see later, is identical with that deduced here for isentropic flow in the channel. Hurrell did not calculate the admittance function, which would have required only modest additional effort.

References 8-10, which report interesting and useful work which we have only recently come to appreciate, fit very closely with our efforts. The representation of the unsteady behavior of the shock described above was used as a boundary condition on a nonuniform subsonic duct. This is essentially the same model we used to treat the inlet of a ramjet engine. ${ }^{3}$ Numerical calculation produced results for both shock position and static pressure, which were in quite good agreement with measurements taken in an externally excited supersonic inlet/subsonic duct model. These conclusions constitute a helpful justification for the view taken here and in our earlier work. ${ }^{3}$

A rather different approach to this problem has been based on more elaborate analysis of two-dimensional transonic flow in channels. Most closely related to the view taken here is the work by Richey and Adamson. ${ }^{11}$ The gencral equation for irrotational compressible flow is expanded in series, using as a small parameter a quantity which measures departures from a uniform stream. Thus the results are limited to cases having relatively weak shock waves; limits on the shock strength are not stated. An example is given for the response of a shock-displacement and velocity-to a sinusoidal disturbance.

The work was later extended 12 to higher amplitude of the shock wave. A particularly interesting feature of these results is the asymmetry of the nonlinear shock motions due to a sinusoidal disturbance. Under certain conditions the shock moves upstream, disappearing as the pressure increases, and then rapidly reappears at its farthermost downstream position as the pressure reaches minimum amplitude. This sort of bchavior has been observed in recent high-speed motion pictures. ${ }^{13}$ The methods developed in Refs. 11 and 12 have been applied also to a problem in which the influences of attached turbulent boundary layers are accounted for..$^{14}$ This is a first step toward treating the more realistic situation in which separation occurs.

The procedure followed here begins in much the same manner as the analyses discussed in Refs. 5 and 6. Apart from some technical differences in the calculations, the main distinction is our intent to develop a representation that fits most easily into an approximate analysis of ramjet engines. For that reason we shall emphasize computation of the admittance function for a steady acoustic wave reflecting from a normal shock wave. The calculation is based on the assumption of quasisteady behavior of the shock wave; the amplitude of the acoustic wave and the amplitude of the motion of the shock wave are assumed small. However, the analysis is also carried out to second order, in order to demonstrate (weakly within the present formulation) the asymmetry of the nonlinear motion mentioned above.

\section{Acoustics of the Inlet}

For the purposes discussed herein we regard a ramjet engine as an inlet section joined to a combustion chamber terminating in a choked exhaust nozzle (Fig. 1) taken from Ref. 3. There is no reason to doubt that the causes of pressure oscillations are processes in the combustion chamber. Wo assume that steady waves are sustained so that the system of acoustic waves in the inlet consists of a wave propagating upstream to the shock, and a reflected wave propagating downstrcam. There is no need here to be concerned with the transmission and reflection of waves at the cnirance to the combustor; it is sufficient to assume that conditions are such as to maintain the steady wave system with wavelength smaller than the length of the inlet. Further consideration of the general problem will appear in a companion paper based on Ref. 3.
We assume further that the properties of the average flow are uniform in the inlet duct, and ignore distributed losses. The governing acoustics equations within the duct where the cross section is constant are the linearized forms of the conservation equations:

$$
\begin{aligned}
& \bar{\rho} \frac{\partial u^{\prime}}{\partial t}+\bar{\rho} \bar{u} \frac{\partial u^{\prime}}{\partial x}+\frac{\partial p^{\prime}}{\partial x}=0 \\
& \frac{\partial p^{\prime}}{\partial t}+\bar{u} \frac{\partial p^{\prime}}{\partial x}+\gamma \bar{p} \frac{\partial u^{\prime}}{\partial x}=0
\end{aligned}
$$

Suitable solutions representing the superposition of rightward (downstream) and leftward (upstream) traveling waves are

$$
\begin{aligned}
& p^{\prime}=\left[P_{+} e^{i k}+x+P_{-} e^{-i k_{-} x}\right] e^{-i \omega t} \\
& u^{\prime}=\left[U_{+} e^{i k_{+} x}+U_{-} e^{-i k_{-} x}\right] e^{-i \omega t}
\end{aligned}
$$

where $+(-)$ denotes the wave traveling to the right (left). Substitution into Eqs. (1) and (2) gives formulas for the wavenumbers and relations among the coefficients.

$$
\begin{aligned}
& k_{+}=\frac{k}{l+\bar{M}} \quad k_{-}=\frac{k}{l-M} \\
& U_{+}=\frac{l}{\bar{\rho} \bar{a}} P_{+} \quad U=\frac{-l}{\bar{\rho} a} P
\end{aligned}
$$

The Mach number of the average flow is $\bar{u} / \bar{a}$ and $k$ is the complex wavenumber relative to the moving stream, $k=(\omega-$ $i \alpha) / \bar{a}$. Eventually, the solutions, Eqs. (3) and (4), may be put in the form

$$
\begin{gathered}
p^{\prime}=\left[P_{+} e^{i K x}+P_{-} e^{-i K x}\right] \exp [-i(\omega t+\bar{M} K x)] \\
u^{\prime}=(I / \bar{\rho} \bar{a})\left[P_{+} e^{i K x}-P_{-} e^{-i K x}\right] \exp [-i(\omega t+\bar{M} K x)]
\end{gathered}
$$

where $K=k /\left(1-\bar{M}^{2}\right)$.

In the diffusion section at the upstream end of the inlet duct, the arca is nonuniform, the shock system resides, and separation may be initiated. It is convenient to represent the influence of the diffuser by an admittance function $A_{0}$. The subscript indicates that it is defined for the plane $x=0$. By definition,

$$
A_{0}=\bar{\rho} \bar{a}\left(u^{\prime} / p^{\prime}\right)
$$

The origin $x=0$ is best taken at the upstream end of the uniform duct; formulas (7) and (8) therefore apply, and substitution into Eq. (9) gives the relation between $P$ and $P_{+}$:

$$
P_{+}=\beta P \quad \beta=\left(I+A_{0}\right) /\left(I-A_{0}\right)
$$

Note, for example, that if $A_{0}$ is real and negative, $\beta<1$ and the amplitude of the reflected wave is less than that of the

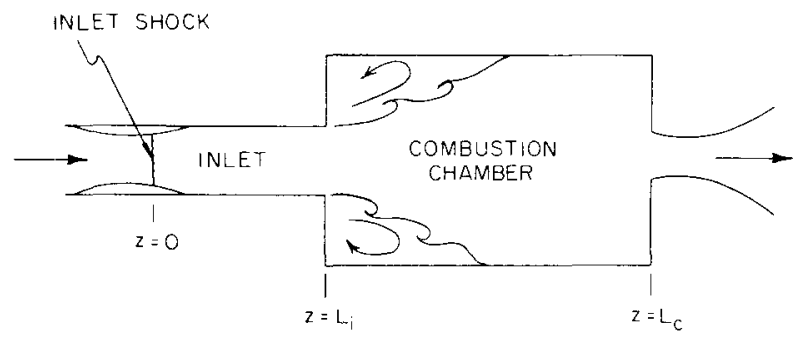

Fig. 1 Sketch of ramjet engine. 
incident wave. The diffuser then acts to attenuate acoustic waves. With Eq. (10), the acoustic pressure and velocity in the uniform section can be written in the useful form

$$
\begin{gathered}
p^{\prime}=P \exp \left[-i\left(\omega t+\bar{M} K x-\psi_{p}\right)\right] \\
u^{\prime}=U \exp \left[-i\left(\omega t+\bar{M} K x-\psi_{u}\right)\right] \\
P=P_{-}\left[1+|\beta|^{2}+2|\beta| \cos (2 K x+\phi)\right] \\
U=(1 / \bar{\rho} \bar{a}) P_{-}[1+|\beta| 2-2|\beta| \cos \phi]^{\prime} \\
\tan \psi_{u}^{\prime}=\frac{-\sin K x+|\beta| \sin (K x+\phi)}{\cos K x+|\beta| \cos (K x+\phi)} \\
\tan \psi_{u}=\frac{\sin K x+|\beta| \sin (K x+\phi)}{-\cos K x+|\beta| \cos (K x+\phi)}
\end{gathered}
$$

The magnitude and phase of $\beta$ follow from Eq. (10):

$$
\begin{gathered}
|\beta|=\left[\frac{\left(1+A_{\theta r}\right)^{2}+A_{\partial i}^{\prime}}{\left(1-\frac{\left.A_{\partial r}\right)^{2}+A_{\partial i}^{2}}{(1)}\right.}\right] \\
\tan \phi=\frac{2 A_{\partial i}}{1-\left(A_{\partial r}^{2}+A_{\partial i}^{2}\right)}
\end{gathered}
$$

These results form the solution for the acoustic field in the inlet duct: it is essentially an impedance tube with an average flow. Because we have neglected distributed losses, the structure of the standing wave field is determined entirely by the complex admittance function representing the action of the diffuser. An important possibility is the inverse casc: If the structure of the wave field is known, Eqs. (11-16) can be used to deduce the value of the complex admittance function. This forms the theoretical basis for an experimental means of determining the influence of the inlet diffuser on pressure oscillations in a ramjet. We shall not pursue the matter here. Our main purpose is to examine the consequences of a simple model for the bchavior of the inlet diffuser to provide a basis for interpreting expcrimental data.

One caveat: must be emphasized. The results deduced in this section are strictly valid only for a uniform duct, with the admittance function $A_{0}$ defined for the upstream entrance plane. The admittance function discussed in the remainder of this paper is that for a shock wave located in the divergent portion of a diffuser, some distance upstream of the entrance to the uniform duct. It may, therefore, be necessary to account for the influence of the short nonuniform region between the shock and the entrance to the inlet duct.

\section{Admittance Function for a Normal Shock in a Diverging Channel}

For application to a one-dimensional model of pressure oscillations in a ramjet, we suppose that any two-dimensional features may, in effect, be averaged over planes transverse to the axis. We consider, therefore, a kind of effective normal shock initially stationary in a diverging duct (Fig. 2). Conditions upstream are supposed to be constant in time. The first problem is to determine the response of the shock to a sinusoidal fluctuation of pressure just downstream of the shock. Motion of the shock produces a reflected wave with associated pressure and velocity fluctuations. The admittance function, $A_{0}$, for the shock is proportional to the ratio of the velocity and pressure fluctuations due to the incident and reflected waves. As we shall emphasize later as well, it is important to realize that the velocity fluctuations in question are only those directly connected to the acoustic pressure fluctuations.
We assume that the frequency of the fluctuations is sufficiently low that the shock responds in a quasisteady fashion. As Burgers 5 first showed, this is a consequence of the rapid gradients of fluid properties through the shock. The assumption of quasisteady behavior means that the flow in the vicinity of the shock satisfies, at each instant of time, the relations which apply if the flow wcre steady with the same conditions applied on both sides of the shock. This implies that the time derivatives may be omitted when solving the general conservation equations. To see that this is a reasonable approximation, consider the equation for conservation of mass,

$$
\frac{\partial \rho}{\partial t}+\frac{\partial}{\partial x}(\rho u)=0
$$

Replace infinitesimals by macroscopic increments; the time scale is the reciprocal of the frequency for significant changes in time, $\Delta t \sim l / f$, and the length scale, $\Delta z$, over which spacial changes are significant, is the shock thickness, $t$. The ratio of the two terms may be estimated as

$$
r=\frac{\partial \rho / \partial t}{\partial(\rho u) / \partial x}-\frac{\Delta \rho / \Delta t}{\Delta(\rho u) / \Delta x} \sim f l \frac{\Delta \rho}{\Delta(\rho u)}
$$

The average density is $\bar{\rho}$, and the average speed $\bar{u}$ is of the order of the spced of sound, so the ratio can be written in terms of fractional changes of the flow properties,

$$
r \sim \frac{f l}{\bar{a}} \frac{(\Delta \rho / \bar{\rho})}{(\Delta(\rho u) / \bar{\rho} \bar{u})}
$$

Now $f / \bar{a}=\lambda$, the wavelength of the acoustic oscillation, and the fractional changes of mean flow properties are all of the same order, so $r \sim p / \lambda$. But the shock thickness is of the order of a few mean free paths, so $r$ is very small; the time derivative may, therefore, be ignored.

The argument may equally be applied to all of the conservation equations, showing that even under unsteady conditions we may, to a good approximation, apply the cquations strictly true for steady flow. Now for steady flow, the solutions to the equations of motion connecting equilibrium states of flow on both sides of the shock are the familiar shock relations. Thus the assumption of quasisteady behavior leads to the result that we may analyze unsteady behavior by substituting into the shock relations the instantaneous values of the flow properties. The problem of unsteady shock motion is, therefore, based on the two formulas:

$$
\begin{aligned}
& \frac{p_{2}}{p_{1}}=I+\frac{2 \gamma}{\gamma+l}\left(M_{l}^{2}-I\right) \\
& \frac{u_{2}}{u_{l}}=I-\frac{2}{\gamma+1}\left(\frac{M_{l}^{2}-I}{M_{l}^{2}}\right)
\end{aligned}
$$

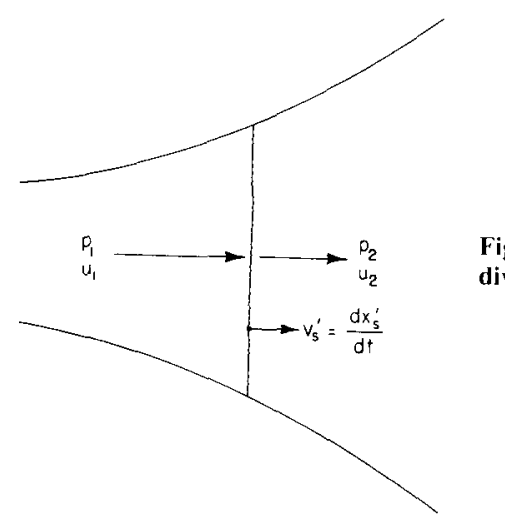

Fig. 2 Normal shock in a diverging channel. 
When the shock moves in response to a downstream disturbance, the upstream conditions fluctuate for two reasons. The shock velocity itself fluctuates, and when it moves it is exposed to a nonuniform flowficld. Thus, the fluctuation of upstream velocity, $u_{/}^{\prime}$, for the conventions defined in Fig. 2, is

$$
u_{i}^{\prime}=-v_{s}^{\prime}+\frac{\mathrm{d} \bar{u}_{I}}{\mathrm{~d} x} x_{s}^{\prime}
$$

where $x_{s}^{\prime}$ is the displacement of the shock from its average position. We retain terms only to first order in small quantities. A fluctuation of the upstream speed of sound is also presented to the shock,

$$
a_{l}^{\prime}=\frac{\mathrm{d} a_{l}}{\mathrm{~d} x} x_{s}^{\prime}
$$

and the associated fluctuation of Mach number is

$$
M_{l}^{\prime}=-\frac{l}{\bar{a}_{l}} v_{s}^{\prime}+\frac{\mathrm{d} \bar{M}_{l}}{\mathrm{~d} x} x_{s}^{\prime}
$$

Finally, the shock experiences the fluctuation of upstream pressure due to its displacement,

$$
p_{i}^{\prime}=\frac{\mathrm{d} \bar{p}_{l}}{\mathrm{~d} x} x_{s}^{\prime}
$$

Similarly, the downstream conditions relative to the shock fluctuate. In addition to the contributions arising from the shock velocity, and its motion into a nonuniform average field, there are fluctuations due to the incident and reflected acoustic waves. Thus, the pressure and velocity fluctuations are represented by the formulas

$$
\begin{gathered}
p_{2}^{\prime}=p_{e}^{\prime}+\frac{\mathrm{d} \bar{p}_{2}}{\mathrm{~d} x} x_{s}^{\prime} \\
u_{2}^{\prime}=-v_{\mathrm{s}}^{\prime}+\frac{\mathrm{d} \bar{u}_{2}}{\mathrm{~d} x} x_{s}^{\prime}+u_{e}^{\prime}
\end{gathered}
$$

According to the assumption of quasisteady behavior, in a reference frame moving with the shock, the jump conditions, Eqs. (19) and (20), must be satisfied at all times. The pressures and velocities are set equal to the average values at the average shock position $\left(x_{s}^{\prime}=0\right)$ plus the fluctuations, Eqs. (21) and $(24-26), p_{l}=\bar{p}_{l}+p_{l}^{\prime}$, etc.

Before carrying through the substitutions, consider the interpretation illustrated in Fig. 3 for the limit $\omega \rightarrow 0$. Line 1 represents the average pressure for isentropic flow through a converging-diverging channel, expanding 10 supersonic Mach numbers. Line 2 represents isentropic diffusion to subsonic flow downstream of the throat. Line 3 is the locus of average pressures, $\bar{p}_{2}$, downstream of a normal shock located within the divergent section. The average pressure for isentropic flow downstream of a typical shock is represented by line 4 .

If the shock $s$ is displaced to $s^{\prime}$, the fluctuations due to motion in the average pressure field are $p_{1}^{\prime}$ and $p_{2}^{\prime}$, as shown. The actual motion of the shock is caused by the fluctuation $p_{c}^{\prime}$, here a reduction of pressure, which because of the assumption of quasisteady behavior must equal the difference between the actual pressure downstream of the shock $s^{\prime}$ in its new position, and the average pressure which would have existed there if the shock had remained at its initial position $s$.

The preceding remarks and Fig. 3 strictly apply only to the limit $\omega=0$. A peculiarity of the analysis is that although the

$\$$ Here and subsequently, derivatives of the average properties are evaluated at the average position of the shock.

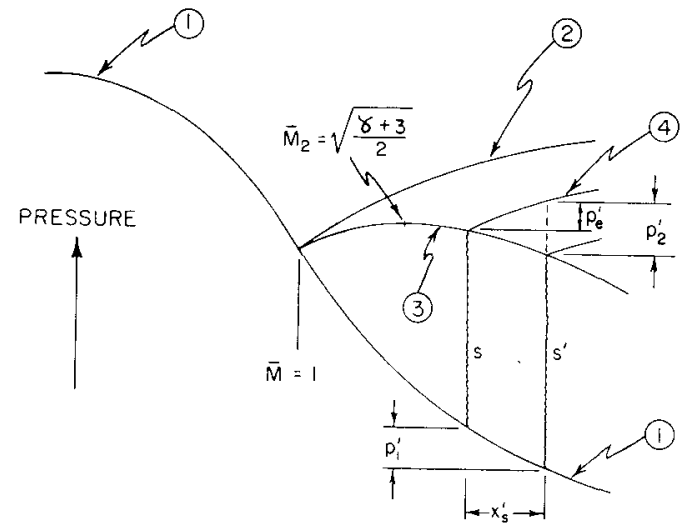

Fig. 3 Average and fluctuating pressure for quasisteady motions in the limit $\omega \rightarrow 0$.

assumption of quasistcady behavior is used throughout, the results exhibit a dependence on frequency. This arises formally because the velocity $v_{s}^{\prime}$ of the shock wave is the time derivative of its displacement, thereby introducing the frequency, $v_{s}^{\prime}=-i \omega x_{s}^{\prime}$ for harmonic motions. This dependence on frequency persists throughout because the discontinuities of pressure and velocity across the shock wave depend on its velocity relative to the upstream flow. Thus, for $\omega>0$, a representation corresponding to Fig. 3 must include adjustments showing the influence of the relative velocity. The qualitative interpretation of the behavior is unchanged.

The fluctuations $p_{e^{\prime}}^{\prime}$ and $u_{e}^{\prime}$ are extcrnally imposed, associated here with the acoustic field, including both the upstream and downstream moving waves. In the definition of the admittance function [Eq. (9)], $p^{\prime}$ and $u^{\prime}$ are, therefore, to be used for $p^{\prime}$ and $u^{\prime}$. Formulas for $p_{e^{\prime}}^{\prime}$ and $u_{e}^{\prime}$ are found by substituting $p_{l}=\bar{p}_{l}+p_{l}^{\prime}$, ctc., with Eqs. (21-26) for the fluctuations, into the shock relations, Eqs. (19) and (20). The results are

$$
\begin{aligned}
& \frac{p_{e^{\prime}}^{\prime}}{\bar{p}_{2}}=-\frac{4 \gamma \bar{M}_{I}}{\gamma+l}\left(\frac{\bar{p}_{l}}{\bar{p}_{2}}\right) \frac{v_{s}^{\prime}}{\bar{a}_{l}}+P_{S}\left(\frac{l}{S} \frac{\mathrm{d} S}{\mathrm{~d} x}\right) x_{s}^{\prime} \\
& u_{c}^{\prime}=\frac{2}{\gamma+l}\left(\frac{\bar{M}_{l}^{2}+1}{\bar{M}_{l}^{2}}\right) v_{s}^{\prime}+\bar{u}_{2} U_{S}\left(\frac{l \mathrm{~d} S}{S} \frac{\mathrm{d} x}{d}\right) x_{s}^{\prime}
\end{aligned}
$$

where $S$ is the cross-sectional area and

$$
\begin{aligned}
& P_{S}=\frac{\mathrm{d} \ln \left(\bar{p}_{1} / p_{2}\right)}{\mathrm{d} l_{1} S}+\frac{2 \gamma}{\gamma+1}\left(\frac{\bar{p}_{1}}{\bar{p}_{2}}\right) \frac{\mathrm{d} \bar{M}_{l}^{2}}{\mathrm{~d} \ln S} \\
& U_{S}=\frac{\mathrm{d} \ln \left(\bar{u}_{1} / \bar{u}_{2}\right)}{\mathrm{d} \ln S}-\frac{4}{\gamma+l}\left(\frac{\bar{u}_{1}}{\bar{u}_{2}}\right) \frac{l}{\bar{M}_{l}^{2}} \frac{\mathrm{d} \ln \bar{M}_{l}}{\mathrm{~d} \ln S}
\end{aligned}
$$

In accordance with the footnote that accompanies Eq. (21), all mean flow properties are evaluated at the average position of the shock.

For the linear problem treated here, we assume steady harmonic motions, so $v_{s}^{\prime}=-i \omega x_{s}^{\prime}$, and substitution of Eqs. (27) and (28) into Eq. (9) with $\bar{\rho} \bar{a}=\bar{\rho}_{2} \hat{a}_{2}$ gives for the admittance function:

$$
\begin{aligned}
A_{0} & =\left[-\frac{2}{\gamma+l}\left(\frac{\bar{M}_{l}^{2}+I}{\bar{M}_{l}^{2}}\right) i \Omega+\bar{M}_{2} U_{\mathrm{S}}\right] \\
& \div\left[\frac{4 \bar{M}_{l}}{\gamma+l}\left(\frac{\bar{p}_{l}}{\bar{p}_{2}}\right)\left(\frac{\bar{a}_{l}}{\bar{a}_{2}}\right) i \Omega+\frac{l}{\gamma} P_{S}\right]
\end{aligned}
$$


The dimensionless frequency $\Omega$ is defined to be

$$
\Omega=\omega / \bar{a}_{2}\left(\frac{I}{S} \frac{\mathrm{d} S}{\mathrm{~d} x}\right)
$$

To see the meaning of $\Omega$, first note that the wavelength of the acoustic oscillations in a gas at rest is $\lambda=2 \pi / k$ $=2 \pi\left(a_{2} / \omega\right)$. Now define a length $L$ such that $(L / S) \mathrm{d} \tilde{S} / \mathrm{d} x=1$; integration gives

$$
S=S_{0} e^{r / l}
$$

and $L$ is the $e$-folding length for the diverging channel. With these definitions, Eq. (32) may be written

$$
\Omega=2 \pi(L / \lambda)
$$

In practice, $\lambda>L$ and $\Omega$ is likely of order one or less; large values of $\Omega$ occur when the length for significant change of area spans at least a substantial fraction of a wavelength.

For a channel having constant area, $P_{S}$ and $U_{S}$ both vanish and the admittance function, Eq. (31), becomes independent of frequency,

$$
A_{0}=-\frac{\left(\bar{M}_{i}^{2}+I\right)}{2(\gamma+l) \bar{M}_{j}^{j}}\left[2 \gamma \bar{M}_{i}^{2}-(\gamma-l)\right] \frac{\bar{a}_{l}}{\bar{a}_{2}}
$$

This is, of course, the limiting case of stability of the shock wave-it is neutrally stable, and there is no preferred position for the shock. Nevertheless, under the action of an oscillating pressure, the displacement of the wave remains finite. For this case, with $v_{s}^{\prime}=-i \omega x_{s}^{\prime}$, Eq. (27) gives

$$
x_{s}^{\prime}=-\frac{i}{\omega} \bar{a}_{1}\left(\frac{\gamma+l}{4 \gamma \bar{M}_{l}}\right) \frac{p_{c}^{\prime}}{\bar{\rho}_{l}}
$$

If the frequency goes to zero, corresponding to a d-c change of pressure from some initial value, the displacement becomes indefinitely large, a reflection of the neutrally stable condition.

The more interesting cases involve flow in a diverging channel, in which a stationary shock is stable. Upstream of the shock wave the flow conditions are well defined and given for the ideal case by the equations for isentropic flow in the nozzle. To be strictly in accord with the assumption of quasisteady behavior and isentropic flow in the nozzle on both sides of the shock, $P_{s}$ and $U_{s}$ must be evaluated using the equations for isentropic flow:

$$
\frac{S \mathrm{~d} \bar{u}}{\bar{u}} \frac{\mathrm{d} x}{\gamma x}=-\frac{I}{\gamma \bar{M}^{2}} \frac{S}{\bar{p}} \frac{\mathrm{d} \bar{p}}{\mathrm{~d} x}=\frac{2}{(\gamma-1) \bar{M}^{2}+2} \frac{S}{\bar{M}} \frac{\mathrm{d} \bar{M}}{\mathrm{~d} x}=\frac{l}{\bar{M}^{2}-1} \frac{\mathrm{d} S}{\mathrm{~d} \bar{x}}
$$

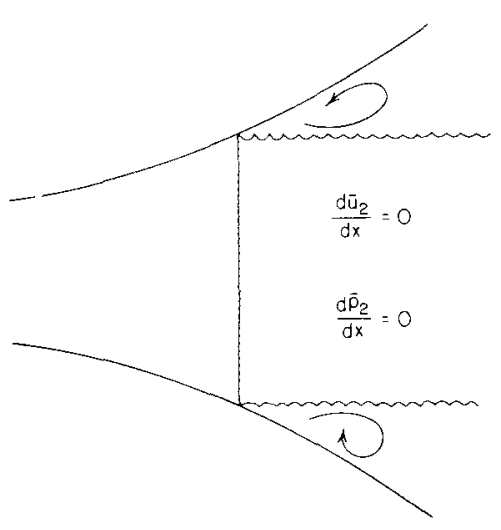

The relation between Mach numbers across a normal shock is also required,

$$
M_{2}^{2}=\frac{2+(\gamma-I) M_{l}^{2}}{2 \gamma M_{l}^{2}-(\gamma-l)}
$$

Substitution into Eqs. (29) and (30) gives

$P_{S}=-\left(\frac{\gamma^{2}+I}{\gamma+I}\right)\left[\bar{M}_{l}^{2}+\frac{\gamma-I}{\gamma^{2}+I}\right] /\left[\bar{M}_{l}^{2}-\frac{\gamma-1}{2 \gamma}\right] ; \quad U_{S}=\frac{2 \gamma}{\gamma+l}$

The admittance function for this case is

$$
\begin{aligned}
& A_{0}=\left[-\frac{2}{\gamma+l}\left(\frac{\bar{M}_{l}^{2}+I}{\bar{M}_{l}^{2}}\right) i \Omega+\frac{2 \gamma}{\gamma+l} \bar{M}_{2}\right]||+\frac{4 \tilde{M}_{l}}{\gamma+l}\left(\frac{\tilde{p}_{l}}{p_{2}}\right) i \Omega \\
& \left.\left.-\frac{l}{\gamma}\left(\frac{\gamma^{2}+1}{\gamma+1}\right) \mid \frac{\bar{M}_{l}^{2}+\left(\frac{\gamma-1}{\gamma^{2}+1}\right)}{\bar{M}_{I}^{2}-\left(\frac{\gamma-1}{2 \gamma}\right)}\right\rceil\right]
\end{aligned}
$$

This result is valid for the ideal case of quasisteady behavior in the limit of inviscid flow. The most significant implication of the second restriction is that no separation occurs. When separation does occur, the flow is twodimensional (at least) and cannot be accommodated by the preceding analysis. As a crude approximation, we suppose that as a consequence of separation, the averaged flow properties downstream of the shock are so affected as to become uniform, as sketched in Fig. 4. We must assume that the separation process responds quickly and begins always at the foot of the shock; moreover, we ignore formation of $\lambda$ shocks which, in fact, are always produced. These assumptions imply that the gradients of downstream properties are zero, so $P_{s}$ and $U_{s}$ are simplified. The admittance function is now

$$
\begin{aligned}
A_{0} & =\left[-\frac{2}{\gamma+I}\left(\frac{\bar{M}_{I}^{2}+I}{\bar{M}_{l}^{2}}\right) i \Omega-\frac{\bar{M}_{2}}{\bar{M}_{I}^{2}-I}\right]||+\frac{4 M_{l}}{\gamma+I}\left(\frac{\bar{p}_{l}}{\bar{p}_{2}}\right) i \Omega \\
& \left.-\frac{l}{\gamma}\left(\frac{\bar{M}_{I}^{2}}{\bar{M}_{I}^{2}-I}\right) \frac{\bar{M}_{I}^{2}-\left(\frac{\gamma+3}{2}\right)}{\bar{M}_{I}^{2}-\left(\frac{\gamma-I}{2 \gamma}\right)}\right] \\
& \text { (approximation to separated flow) }
\end{aligned}
$$

The real and imaginary parts of the admittance functions are shown in Fig. 5 for inviscid nozzle flow and in Fig. 7 for the approximation to separated flow. Figures 6 and 8 show the results for the reflection coefficient, $|\beta|$. To obtain numerical values for use in analysis of an engine, it is necessary to specify the Mach number of the shock, the frequency, the speed of sound just downstream of the shock, and the gradient of the cross-sectional area.

When the admittance function is incorporated as a boundary condition in analysis of the stability of acoustics, it happens that the imaginary part, $A_{i}$, causes a shift of the frequency, and the damping of the waves is proportional to the real part: as defined here, waves are attenuated-the shock wave acts to dissipate acoustic energy - when the real part is negative. According to the results obtained here, for unseparated (isentropic) nozzle flow, the shock wave always 


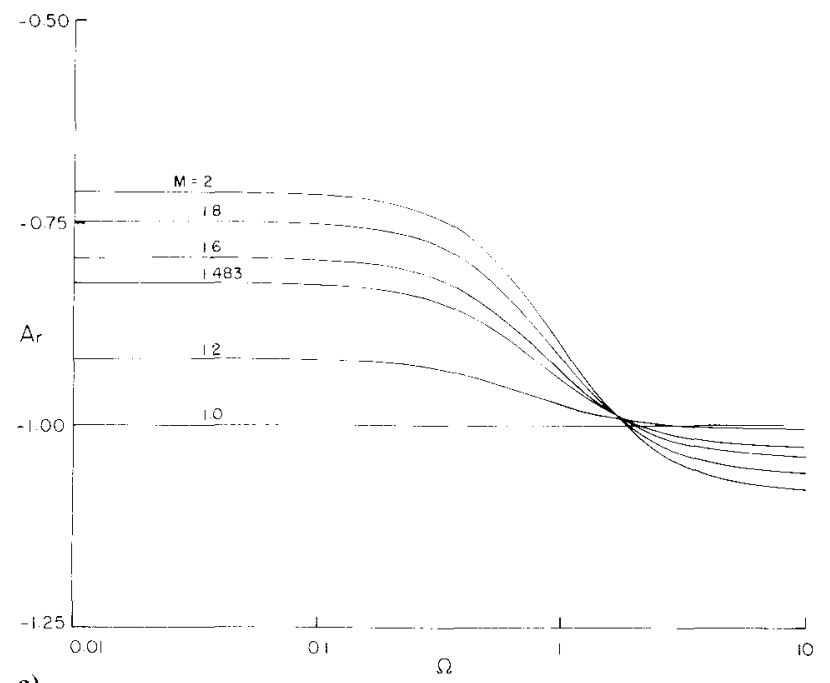

a)
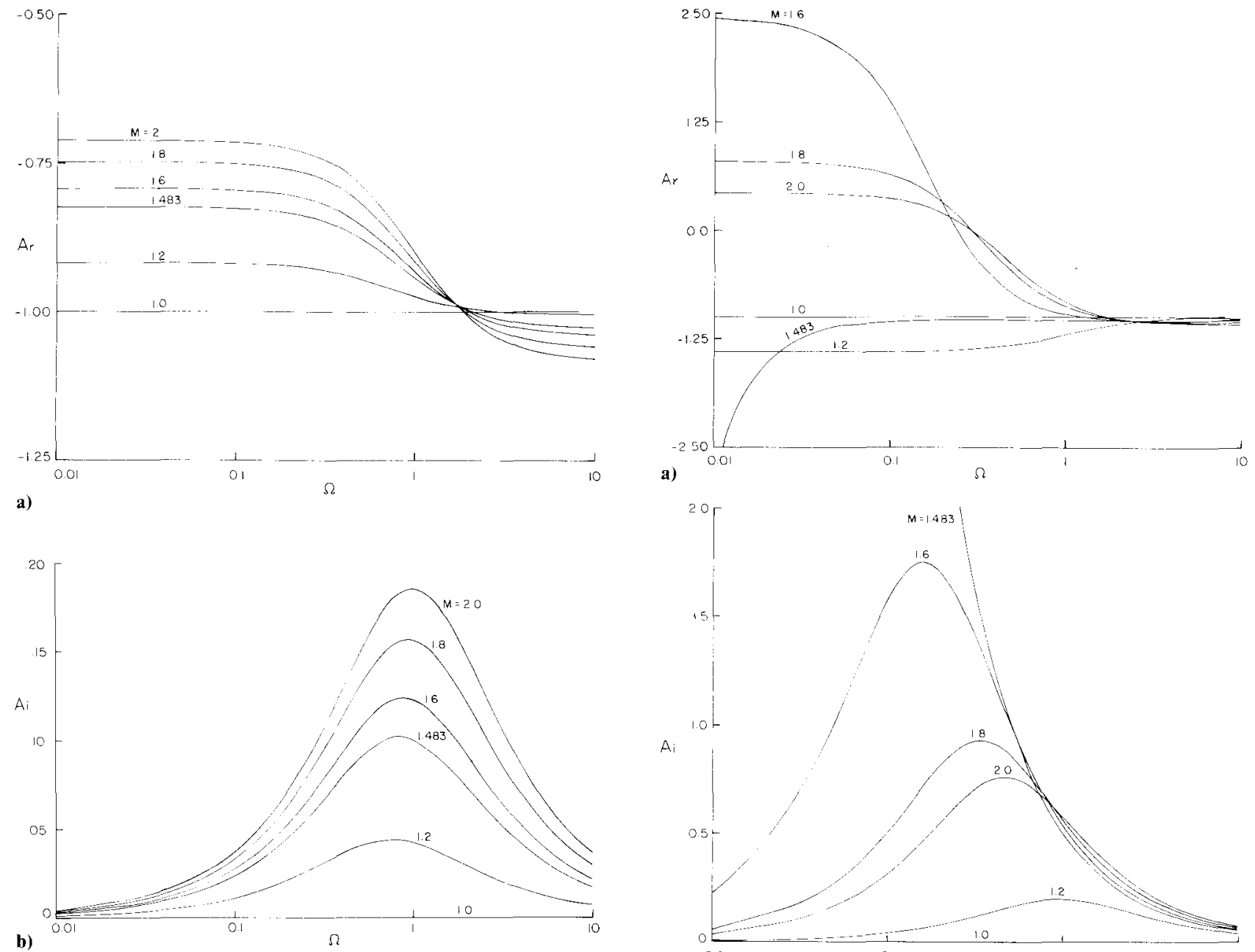

Fig. 5 Complex admittance function for isentropic nozzle flow. a) real part; b) imaginary part.

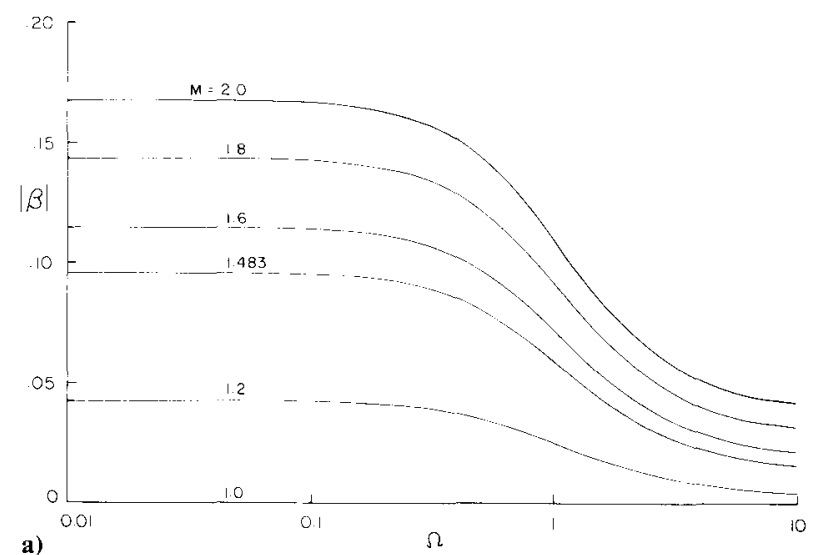

b)

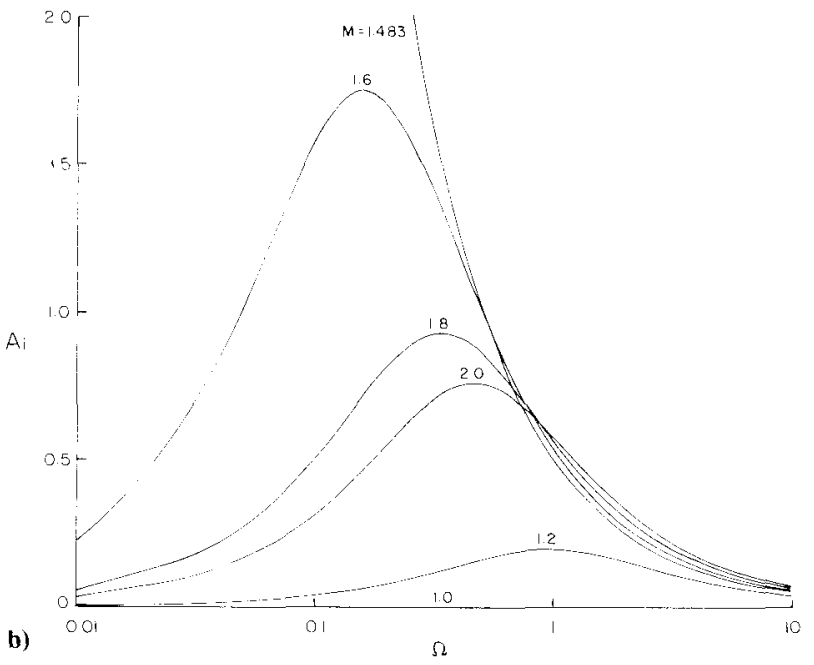

Fig. 7 Complex admittance function for the approximation to separated flow. a) real part; b) imaginary part.

attenuates oscillations: $A_{r}$, Fig. $5 \mathrm{a}$, is never positive. On the contrary, according to Fig. 7a, for the approximation to separated flow, there is evidently a rather broad range of lower frequencies, and a higher Mach number in which the shock wave may act to drive the oscillations. The computations in the next section show that the shock itself is stable only if $M_{i}^{2}<(\gamma+3) / 2$, so only in that rangc can these results be used. Correspondingly, the real part of Eq. (40) is positive for $M_{7}^{2}>(\gamma+3) / 2$.

\section{Stability of a Normal Shock Wave}

The displacement of the shock wave may be calculated from $\mathrm{Eq} .(27)$ :

$$
x_{s}^{\prime}=\frac{p_{e}^{\prime}}{\bar{p}_{2}} /\left[-\frac{4 \gamma \bar{M}_{1}}{\gamma+l}\left(\frac{\bar{p}_{l}}{\bar{p}_{2}}\right) i \frac{\omega}{\bar{a}_{2}}+P_{S}\left(\frac{l}{S} \frac{\mathrm{d} S}{\mathrm{~d} x}\right)\right]
$$

With the formulas for isentropic flow, this can be put in the form

$$
\frac{x_{s}^{\prime}}{p_{e}^{\prime}}=\frac{C}{1-i \omega \tau}
$$

with

$$
C=-\frac{I}{\bar{p}_{l}}\left(\frac{1}{S} \frac{\mathrm{d} S}{\mathrm{~d} x}\right)^{-1} \frac{(\gamma+1)^{2}}{2 \gamma(\gamma-I)\left(1+\frac{\gamma^{2}+1}{\gamma-1} \bar{M}_{l}^{2}\right)}
$$

Fig. 6 Reflection coefficient, isentropic flow. a) magnitude; b) phase. 


$$
\tau=\frac{1}{\bar{a}_{I}}\left(\frac{1}{S} \frac{\mathrm{d} S}{\mathrm{~d} x}\right)^{-1} \frac{2(\gamma+1) \bar{M}_{1}}{(\gamma-1)\left(1+\frac{\gamma^{2}+1}{\gamma-l} \bar{M}_{l}^{2}\right)}
$$

Formulas (42-44) agree exactly with the result given by Hurrel17; it is the solution for the motion of the shock when the pressure fluctuation is sinusoidal. In general, the equation for linear motion of the shock is, therefore,

$$
\frac{\mathrm{d} x_{s}^{\prime}}{\mathrm{d} t}+\frac{l}{\tau} x_{s}^{\prime}=\frac{C}{\tau} p_{\prime}^{\prime}
$$

That a normal shock is stable in a diverging channel and unstable in a converging channel follows directly. A simple way to show this is to consider the general solution

$$
x_{s}^{\prime}(t)=\frac{C}{\tau} e^{-t / \tau} \int_{0}^{\prime} e^{t^{\prime} / \tau} p_{e}^{\prime}\left(t^{\prime}\right) \mathrm{d} t^{\prime}
$$

where we have required $x_{s}^{\prime}=0$ at $t=0$. Suppose, for example, that the pressure disturbance is nonzero only for a finite length of time. Then, assuming that it exists, as it must for realizable disturbances, the integral in Eq. (46) is just a number, independent of time. Hence, the displacement decays to zero for large times if $\tau$ is positive, and grows indefinitely large if $\tau$ is negative. The first case is stable and, according to Eq. (44), occurs for a diverging channel, $\mathrm{d} S / \mathrm{d} x>0$. This answers the question of stability, subject only to the assumption of quasisteady behavior of the shock and isentropic flow in the nozzle.

For the approximation to separated flow,

$P_{S}=-\left[\bar{M}_{l}^{2}\left(\bar{M}_{l}^{2}-\frac{\gamma+3}{2}\right)\right] /\left[\left(\bar{M}_{I}^{2}-l\right)\left(\bar{M}_{I}^{2}-\frac{\gamma-I}{2}\right)\right]$

By comparison of Eqs. (41) and (42) we find the formula for the relaxation time $\tau$,

$$
\tau=-\frac{I}{\bar{a}_{1} P_{s}}\left(\frac{1}{S} \frac{\mathrm{d} S}{\mathrm{~d} x}\right)^{-1}\left(\frac{\bar{p}_{1}}{\bar{p}_{2}}\right) \frac{4 \gamma \bar{M}}{\gamma+1}
$$

The shock wave is stable only if $\tau$ is positive, which requires $P_{S}$ to be negative for a diverging channel. From Eq. (47), $P_{S}$ is negative only if $\bar{M}_{1}^{2}<(\gamma+3) / 2$. As shown in Fig. 3, this is the Mach number at which the pressure downstream of the shock passes through a maximum value.

This result is interesting, showing that the stability of a shock wave in a diverging channel is not always assured. Obviously, the admittance function computed above cannot be used for Mach numbers less than the value at which the shock is unstable. Whether the instability of the shock at the lower Mach numbers in the presence of separation is a real phenomenon remains to be seen. The physical origin of the instability arises from the reduced pressure recovery due to the separated region downstream of the shock. Even though $\mathrm{d} p_{2} / \mathrm{d} x$ may not actually vanish as assumed here, the result suggests that the stability of normal shock waves will be unfavorably influenced by separation. The reduction of stability may be compensated in some way by the formation of $\lambda$ shocks, but the problem of the stability of nonplanar shocks in a channel seems not to have been examined.

\section{Nonlinear Motion of a Shock Wave}

Nonlinear motion of a shock may be examined in straightforward fashion within the framework constructed here. We continue to assume quasisteady behavior, but now carry out the approximation to second order in small quantities. Because the shock relations, Eqs. (19) and (20), apply, the instantaneous properties satisfy

$$
\begin{gathered}
\frac{p_{2}(\xi, l)}{p(\xi)}=1+\frac{2 \gamma}{\gamma+1}\left[\left\{M(\xi) \frac{\dot{\xi}}{a_{l}(\xi)}\right\}^{2}-1\right] \\
\frac{u_{2}(\xi, t)-\dot{\xi}}{u(\xi)-\dot{\xi}}=1-\frac{2}{\gamma+1} \frac{\left\{M_{l}(\xi)-\frac{\dot{\xi}}{a_{l}(\xi)}\right\}^{2}-1}{\left\{M_{l}(\xi)-\frac{\dot{\xi}}{a_{l}(\xi)}\right\}^{2}}
\end{gathered}
$$

For simplicity, we now write $\xi=x_{s}^{\prime}$ and $\dot{\xi}=v_{s}$. The procedure now consists in expanding all variables to second order in $\xi, \dot{\xi}$, and the applied fluctuations; thus, for example,

$$
\begin{aligned}
& p_{1}(\xi)=\bar{p}_{1}(0)+\left(\frac{\mathrm{d} \bar{p}}{\mathrm{~d} x}\right)_{0} \xi+\frac{l}{2}\left(\frac{\mathrm{d}^{2} p_{1}}{\mathrm{~d} x^{2}}\right)_{0} \xi^{2} \\
& p_{2}(\xi, t)=\bar{p}_{2}(0)+\left(\frac{\mathrm{d} p_{2}}{\mathrm{~d} x}\right)_{0} \xi+\frac{1}{2}\left(\frac{\mathrm{d}^{2} \bar{p}_{2}}{\mathrm{~d} x^{2}}\right)_{0} \xi^{2} \\
& \quad \cdot \quad+p_{2}^{\prime}(0, t)+\left(\frac{\partial p_{2}^{\prime}}{\partial \mathrm{d} x}\right)_{0} \xi \\
& \quad \cdot \quad \\
& \quad \cdot \quad \\
& \text { etc. }
\end{aligned}
$$

where subscript ( ) denotes values at $x=\xi=0$. After all the arithmetic is completed, Eqs. (48) and (49) lead to the forms

$$
p_{2}^{\prime}(0, t)+\left(\frac{\partial p_{2}^{\prime}}{\partial x}\right)_{0} \xi=P_{1} \xi+P_{2} \dot{\xi}+P_{3} \xi^{2}+P_{4} \xi^{2}+P_{5} \xi \dot{\xi}
$$

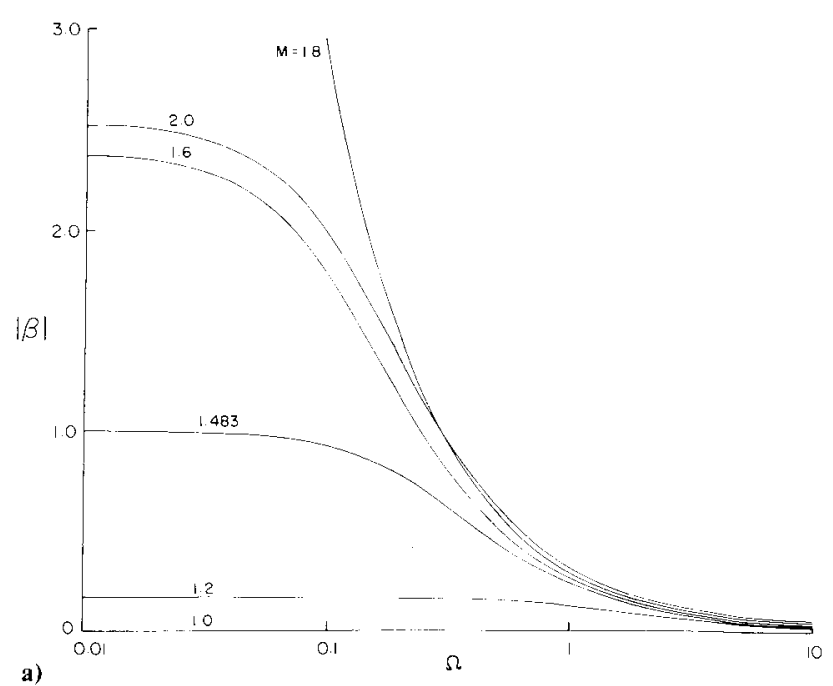

a)

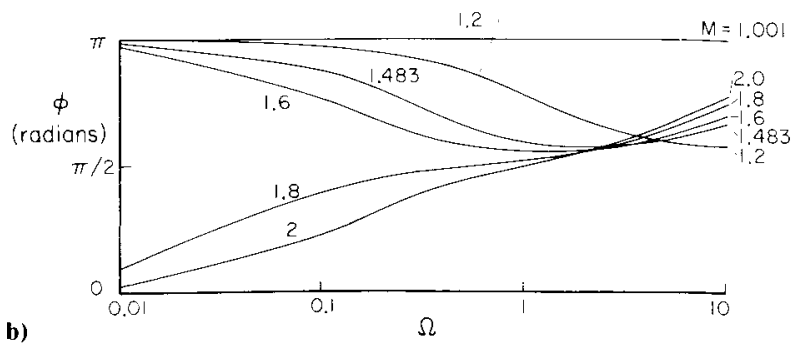

Fig. 8 Reflection coefficient, separated flow. a) magnitude; b) phase. 


$$
u_{2}^{\prime}(0, t)+\left(\frac{\partial u_{2}^{\prime}}{\partial x}\right)_{0} \xi=U_{1} \xi+U_{2} \dot{\xi}+U_{3} \xi^{2}+U_{4} \dot{\xi}^{2}+U_{5} \xi \dot{\xi}
$$

These reduce to the linearized results, Eqs. (27) and (28), upon dropping all terms of second order, including the derivatives on the left-hand sides.

It is now not meaningful to assume sinusoidal motions, and the admittance function is not a useful quantity. These equations could be used to formulate a boundary condition for analysis of second-order acoustics in the inlet, but here we consider only the nonlinear motion of the shock in the presence of a specified varying pressure. This rests on the solution of Eq. (50) for $\xi$. We shall treat only the simplest example, to demonstrate the asymmetry of motion cited in the Introduction. The most direct way to the result is by an iterative procedure, which we shall carry out only through the second approximation.

Write Eq. (50) as

$$
\dot{\xi}+c \xi=\frac{l}{P_{2}} p_{2}^{\prime}(0, t)+\frac{1}{P_{2}}\left[\left(\frac{\partial p_{2}^{\prime}}{\partial x}\right)_{0} \xi-P_{3} \xi^{2}-P_{4} \dot{\xi}^{2}-P_{5} \xi \dot{\xi}\right]
$$

where $c=P_{1} / P_{2}$. The nonlinear terms, ignored in the first approximation, are enclosed in brackets. For linearized motions the solution is Eq. (46); in the present notation, with a nonzero value $\xi_{l}(0)$ of $\xi_{l}$ at $t=0$,

$$
\xi_{1}(t)=\frac{l}{P_{2}} e^{-c t} \int_{0}^{t} e^{c t} p_{2}^{\prime}(0, t) \mathrm{d} t^{\prime}+\xi_{1}(0) e^{-c t}
$$

The second approximation is the solution to Eq. (52) with $\xi$, substituted in the nonlinear terms:

$$
\begin{aligned}
& \xi_{2}(t)=\frac{l}{P_{2}} e^{-t t} \int_{0}^{t} e^{c t^{\prime}} p_{2}\left(0, t^{\prime}\right) \mathrm{d} t^{\prime}+\xi_{1}(0) e^{-c t} \\
& +\frac{l}{P_{2}} e^{-c t} \int_{0}^{t} e^{c t^{\prime}}\left[\left(\frac{\partial p_{2}^{\prime}}{\mathrm{d} x}\right)_{0} \xi-P_{3} \xi_{l}^{2}-P_{4} \dot{\xi}_{l}^{2}-P_{5} \xi_{1} \dot{\xi}_{l}\right] \mathrm{d} t^{\prime}
\end{aligned}
$$

Rather than continue with this general form, the main point can be made using the motion in a uniform duct as an example. For that case, the coefficients $P_{1}, \ldots, P_{5}$ are

$$
P_{1}=P_{3}=P_{5}=0 ; \quad P_{2}=-\frac{4 \gamma \bar{p}_{l} \bar{M}_{1}}{(\gamma+l) \bar{a}_{l}} ; \quad P=\frac{2 \gamma \bar{p}_{1}}{(\gamma+l) \bar{a}_{l}^{2}}
$$

Assume also that $p_{2}^{\prime}(x, t)$ is uniform in space, having amplitude $\epsilon \bar{p}$.

$$
p_{2}^{\prime}=\epsilon \bar{p} \cos \omega t
$$

Formula (54) simplifies to

$$
\xi_{2}(t)=\xi_{l}(t)+\frac{1}{2 \bar{u}_{l}} \int_{0}^{t} \dot{\xi}_{l}^{2} \mathrm{~d} t^{\prime}
$$

and the velocity to second order is

$$
\frac{\mathrm{d} \xi_{2}}{\mathrm{~d} t}=\frac{\mathrm{d} \xi_{1}}{\mathrm{~d} t}+\frac{l}{2 \bar{u}_{1}}\left(\frac{\mathrm{d} \xi_{1}}{\mathrm{~d} t}\right)^{2}
$$

The linear approximation to the velocity in this case is

$$
\frac{\mathrm{d} \xi_{I}}{\mathrm{~d} t}=-\frac{\bar{a}_{1}}{2 \bar{M}_{l}}\left(\frac{\gamma+l}{2 \gamma}\right) \frac{p_{2}^{\prime}}{\bar{p}_{l}}
$$

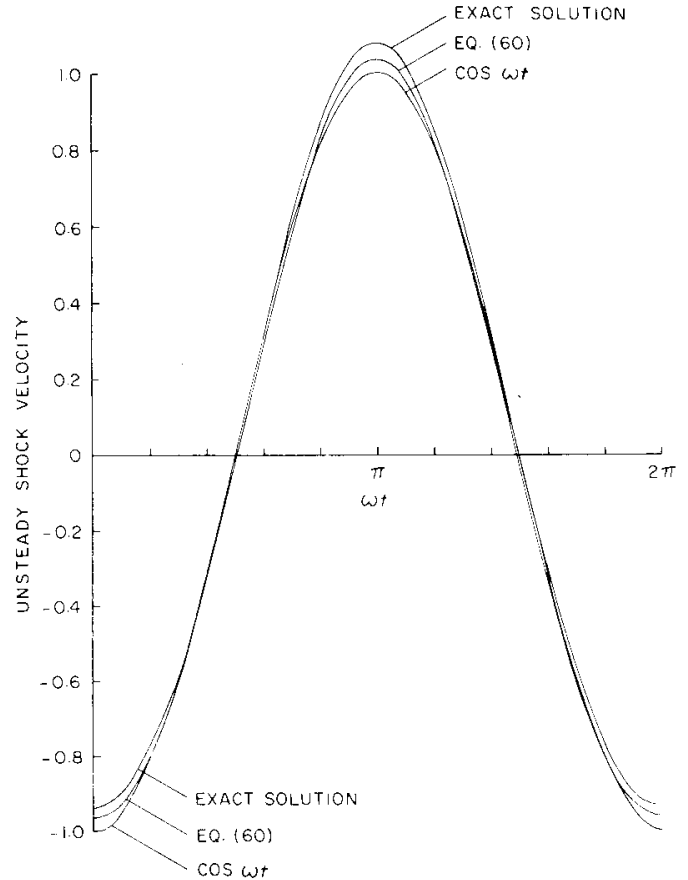

Fig. 9 Velocity of a shock in a uniform channel, with a sinusoida pressure disturbance $\left(\vec{M}_{1}=1.25,\left|p^{\prime} / \bar{p}\right|=0.5\right)$.

and Eq. (58) can be written

$$
\frac{4 \gamma \bar{M}_{l}}{(\gamma+I) \bar{a}_{l}} \frac{\mathrm{d} \xi_{2}}{\mathrm{~d} t}=-\frac{p_{2}^{\prime}}{\bar{p}_{l}}+\left(\frac{\gamma+I}{c \gamma}\right) \frac{I}{\bar{M}_{l}^{2}}\left(\frac{p_{2}^{\prime}}{\bar{p}_{l}}\right)^{2}
$$

With Eq. (56), the shock velocity may finally be expressed as

$$
\frac{l}{\epsilon} \frac{4 \gamma \bar{M}_{1}}{(\gamma+1) \bar{a}_{l}} \frac{\mathrm{d} \xi_{2}}{\mathrm{~d} t}=-(\cos \omega t-\Delta \cos 2 \omega t)+\Delta
$$

where the influence of nonlinear behavior is measured by

$$
\Delta=\left(\frac{\gamma+l}{16 \gamma}\right) \frac{\epsilon}{\bar{M}_{l}^{2}}
$$

It is a curious result that in addition to the distortion at twice the imposed frequency, the nonlinearities cause the shock to move downstream rather than upstream at the steady rate proportional to $\Delta$.

Figure 9 shows the oscillatory part of the velocity, comparing the linear and nonlinear results. Note that the peak velocity during motion downstream is increased, while the negative velocity is reduced in a region about its maximum value. This is suggestive of observations mentioned earlier, but the problem of nonlinear motion in a diverging channel should be analyzed to provide a closer approximation.

\section{Concluding Remarks}

The model treated here is the simplest possible representation of the unsteady behavior of the inlet shock system. It should be regarded as a means of treating the processes averaged in some sense over the cross section of the channel. While evidently crude, the results are convenient for use in

It is easily shown that this is the second-order approximation to the exact solution for this case

$$
\frac{l}{\bar{u}_{l}} \frac{\mathrm{d} \xi}{\mathrm{d} t}=1-\left[l+\left(\frac{\gamma+l}{2 \gamma}\right) \frac{l}{\bar{M}_{l}^{2}} \frac{p_{2}^{\prime}}{\bar{p}_{l}}\right]^{1 / 2}
$$


one-dimensional analysis of pressure oscillations in the inlet or in the entire engine, and as a basis for interpreting experimental results. It is particularly important to emphasize that the analysis encompasses the acoustic field only. Velocity fluctuations which may arise, for example, due to separation and which do not propagate with the speed of sound have not been accommodated.

\section{In Memoriam}

On February 7, 1981, while this work was in progress, Tom Rogers suffered a fatal heart attack while engaged in his favorite sport, cycling. The profession has lost an unusually talented and highly respected member; I have lost a close friend.

\section{Acknowledgments}

I am indebted to Dr. M. Sajben for several useful discussions, particularly concerning the approximation of quasisteaciy behavior, and to V. Yang for his help in completing some of the calculations. This work was supported partly by the Navy Liquid Fuel Ramjet Component Technology Program (N00123-77-C-0541) and partly by the Air Force Office of Scientific Research, Grant AFOSR-800265 .

\section{References}

${ }^{1}$ Rogers, T., "Ramjet Inlet/Combustor Pulsations Study," Naval Weapons Center Rept. NWC TP 6053, Jan. 1980.
${ }^{2}$ Rogers, T., "Ramjet Inlet/Combustor Pulsations Analysis Test," Naval Weapons Center Rept. NWC TP 6155, Feb. 1980.

${ }^{3}$ Culick, F.E.C. and Rogers, T., "Modeling Pressure Oscillations in Ramjet Engines," AIA Paper 80-1192, June 1980, pp. 80-1117.

${ }^{4}$ Clark, W. H., "An Experimental Investigation of Pressure Oscillations in a Side Dump Ramjet Combustor," AIAA Paper 801117, June 1980.

${ }^{5}$ Burgers, J. M., "On the Transmission of Sound Waves through a Shock Wave," Proceedings, Koninklijke Nederlandse Akademie van Wetenshappen, Vol. 49, No. 3, 1946, pp. 274-281.

${ }^{6}$ Kantrowitz, A., "The Formation and Stability of Normal Shock Waves in Channel Flows," NACA TN 1225, Nov. 1946.

${ }^{7}$ Hurrell, H. G., "Analysis of Shock Motion in Ducts During Disturbances in Downstream Pressure," NACA TN 4090, July 1957.

${ }^{8}$ Willoh, R. G., "A Mathematical Analysis of Supersonic Inlet Dynamics," NASA TND-4969, Aug. 1968.

${ }^{9}$ Wasserbauer, J. F. and Whipple, D. L., "Experimental Investigation of the Dynamic Response of a Supersonic Inlet to External and Internal Disturbances," NASA TMX-1648, Sept. 1968.

${ }^{10}$ Wasserbauer, J. F. and Walloh, R. G., "Experimental and Analytical Investigation of the Dynamic Response of a Supersonic Mixed Compression Inlet," AIAA Paper 68-651, AIAA 4th Propulsion Joint Specialist Conference, June 1968.

${ }^{" R i c h e y, ~ G . ~ K . ~ a n d ~ A d a m s o n, ~ J r . ~ T . ~ C ., ~ " A n a l y s i s ~ o f ~ U n s t e a d y ~}$ Transonic Channel Flow with Shock Waves," AIAA Journal, Vol. 14, Aug. 1976, pp. 1054-1061.

${ }^{12}$ Adamson, T. C, Messiter, A. F, and Liou, M. S., "L Large Amplitude Motion in Two-Dimensional Transonic Channel Flows," AIA A Journal, Vol. 16, Dec. 1978, pp. 1240-1247.

${ }^{13}$ Schadow, K., private communication, Naval Weapons Center, China Lake, Calif., Aug. 1981.

${ }^{14}$ Liou, M. S. and Sajben, M., "Analysis of Unsteady Viscous Transonic Flow with a Shock Wave in a Two-Dimensional Channel," AlAA Paper 80-0195, 18th AIAA Aerospace Sciences Mecting, Jan. 1980. 